\title{
PERFORMANCE ANALYSIS OF CNTFET AND MOSFET FOCUSING CHANNEL LENGTH, CARRIER MOBILITY AND BALLISTIC CONDUCTION IN HIGH SPEED SWITCHING
}

\author{
Md. Alamgir Kabir*, Turja Nandy, Mohammad Aminul Haque, Arin Dutta, Zahid \\ Hasan Mahmood \\ Applied Physics, Electronics and Communication Engineering \\ University of Dhaka \\ Dhaka-1000, Bangladesh
}

\begin{abstract}
Enhancement of switching in nanoelectronics, Carbon Nano Tube (CNT) could be utilized in nanoscaled Metal Oxide Semiconductor Field Effect Transistor (MOSFET). In this review, we present an in depth discussion of performances Carbon Nanotube Field Effect Transistor (CNTFET) and its significance in nanoelectronic circuitry in comparison with Metal Oxide Semiconductor Field Effect Transistor (MOSFET). At first, we have discussed the structural unit of Carbon Nanotube and characteristic electrical behaviors beteween CNTFET and MOSFET. Short channel effect and effects of scattering and electric field on mobility of CNTFET and MOSFET have also been discussed. Besides, the nature of ballistic transport and profound impact of gate capacitance along with dielectric constant on transconductance have also have been overviewed. Electron ballistic transport would be the key in short channel regime for high speed switching devices. Finally, a comparative study on the characteristics of contact resistance over switching capacity between CNTFET and MOSFET has been addressed.
\end{abstract}

\section{KEYWORDS}

CNTFET, MOSFET, schottky barrier, channel length, carrier mobility, MFP, SCE, DIBL, transconductance, gate capacitance, ballistic conduction, contact resistance.

\section{INTRODUCTION}

Semiconductor based technology has gone through a tremendous advancement in recent decades. In the field of manufacturing integrated circuit, Moore's law dominates the evolution in the number as well as the size of transistors. But in nanoscale fabrication, field effect transistors have been suffered from direct source-drain tunneling because of short channel effects [1]. As MOS infrastructure continues to scale down deeper into the nanoscale, various non-ideal characteristics, substantially different from MOSFET are aroused. Carbon Nanotube (CNT), the rolled structure of graphene, renders itself to multi-disciplinary applications in nanoelectronic circuits and removes those effects [2]. The field effect transistors consisting of CNT are called 
CNTFET and recently they have attracted the attention as possible building blocks of future nanoelectronics due to their distinctive structural, electrical and mechanical properties [3]. These provide notable characteristics over MOSFET using the semiconducting properties of CNT like high carrier mobility, large MFP (mean free path) [4], ballistic conduction [5], suitable contact resistance [6], fast switching speed and less heat dissipation [6, 7]. Carbon nanotube based transistors can work as a molecular electronic device in terahertz region in comparison with silicon based transistors.

Although CNT has less reliability and greater cost problem, it can play a vital role in nanoelectronic devices such as transistors, memory components $[8,9]$ etc. because of its excellent electrical conductivity and high dielectric properties [10, 11]. Therefore, Field Effect Transistors have been tried to be constructed from CNT to surmount the limitations of Si-MOSFET such as carrier mobility, channel length [8], ballistic conduction, transconductance [9, 10], heat dissipation, threshold voltage etc. [10-12].

\section{CARBON NANOTUBE (CNT) \& CARBON NANOTUBE FIELD EFFECT TRANSISTOR (CNTFET)}

CNT is a carbon allotropic variety which comes from the fullerenes family. It is made of nano sized carbon atom and rolled in seamless cylindrical form of single atomic layered thick grapheme sheet [13]. Fig. 1 (a) shows a CNT which is made from one sheet of graphene rolled in cylindrical structure which is called Single Wall Carbon Nanotube (SWCNT) and Fig. 1 (b) shows Multi Walled Carbon Nanotube (MWCNT) where multi-layers of graphene are rolled as a concentric tube [14-16].

CNT based nano devices are comprised of wide range of nano structures. CNTFET could be used as valve or controlled switch in electronics. Carbon Nano Tube Field Effect Transistors for NEMS are emerging day by day not only in IC industry but also in medical science, mechanical system, automobile industry and recreational instruments [17].

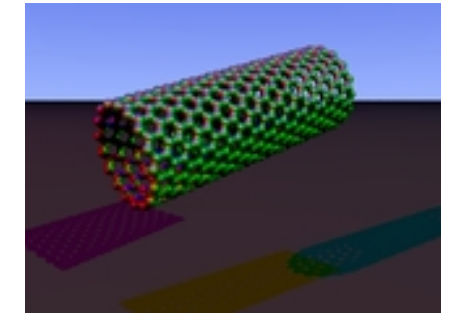

(a)

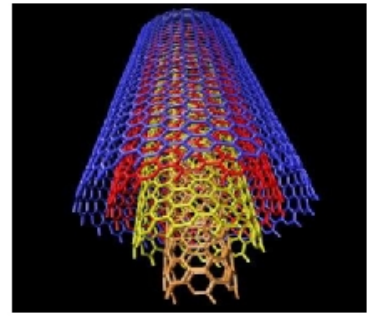

(b)

Figure 1 (a). SWCNT and (b) MWCNT [14, 15].

CNT has two natures, metallic and semiconducting. The semiconducting natures of CNT is used to manufacture CNTFET device. For the property of high conductivity, SWCNT is being largely used in CNTFET fabrication [16]. 


\section{CNTFET PERFORMANCES OVER MOSFET PERFORMANCES}

CNTFET has distinct nanoscale properties that has been absent in MOSFET microelectronics. Thus MOS technology is not promising alternative for fabricating dynamic nano devices. CNT could be used in nanoscaled MOSFET in gate region over dielectric substrate. So, that gate oxide has little effect in CNTFET than MOSFET. Fig. 2 (a) shows the schematic view of CNTFET and Fig. 2 (b) the same view of MOSFET. Circuit performance in CNTFET and MOSFET, we need drain current against drain voltage curve (I-V) for various gate voltage which is shown in Fig. 3 . There are linear curve region in MOSFET curve which is not available in CNTFET, which has gradual saturation region providing better electrical behavior than MOSFET.

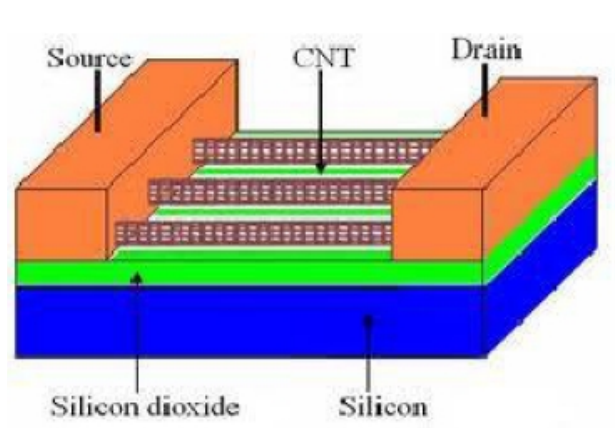

(a)

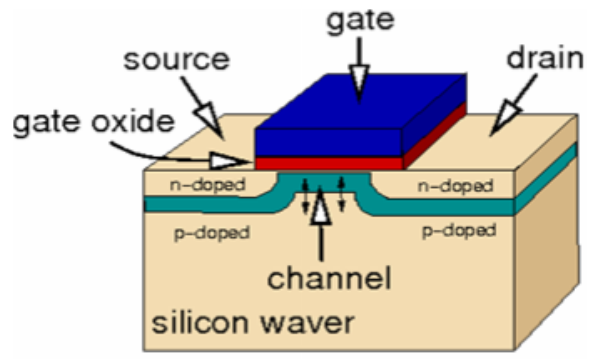

(b)

Figure 2. (a) Carbon Nanotube Field Effect Transistor (CNTFET) [18] and (b). Metal-OxideSemiconductor Field Effect Transistor (MOSFET) [19]

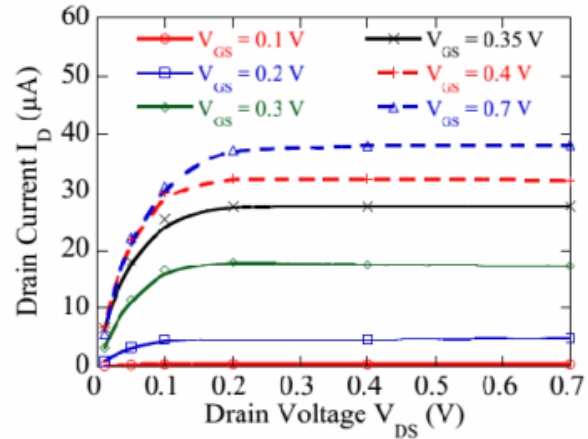

(a)

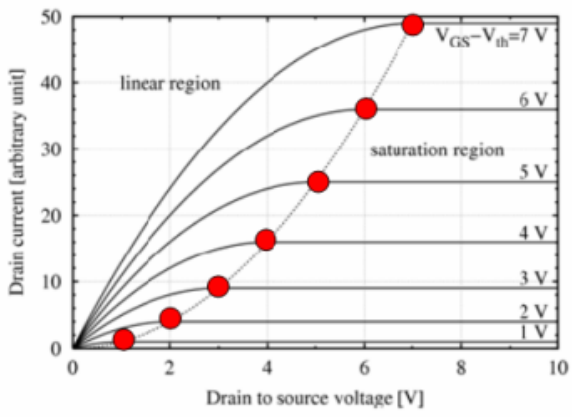

(b)

Figure 3 (a) \& (b). I-V characteristics (Drain current vs. Drain voltage) for different gate voltage of CNTFET and MOSFET respectively [20, 21].

\subsection{High carrier mobility over long channel length}

At CNTFETs, having long channel length, the mobility of carriers is high. Fig. 4 (a) shows that $\mathrm{SiO} 2$ provides the better path for the electronic transport between the electronic channel and 
dielectric layer as compared to other high- dielectrics. When channel length of CNTFET is modified with chemical etching, this effect is seen which increases the mobility of carriers. But in case of MOSFET, due to some scattering mechanisms, such as phonon scattering, surface roughness scattering, columbic scattering from interface, bulk charge impurity scattering etc., the mobility of electrons become affected. Although, Fig. 4 (b) shows, it also provides electric transport for $\mathrm{SiO} 2$ dielectric layer which is nearly identical to CNTFET [22-25].

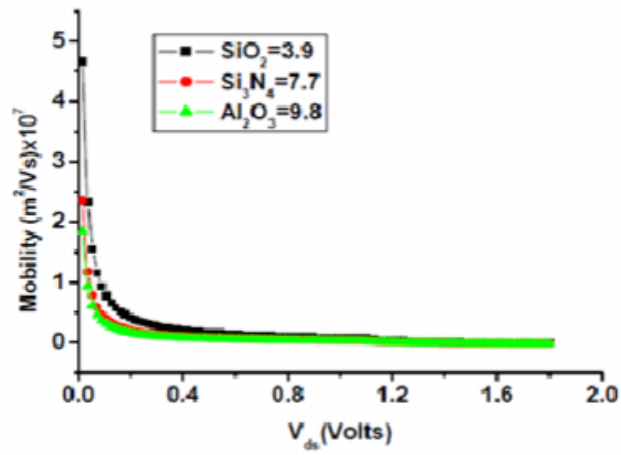

(a)

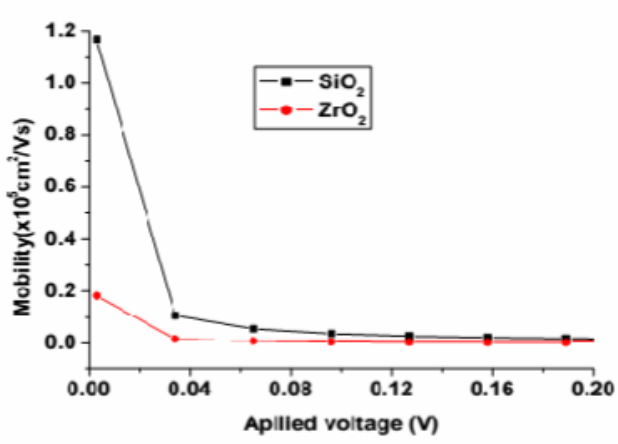

(b)

Figure 4 (a). Variation of mobility for different channel lengths (micrometer) of CNTFET and (b). Variation in mobility with different dielectric material in MOSFET [25].

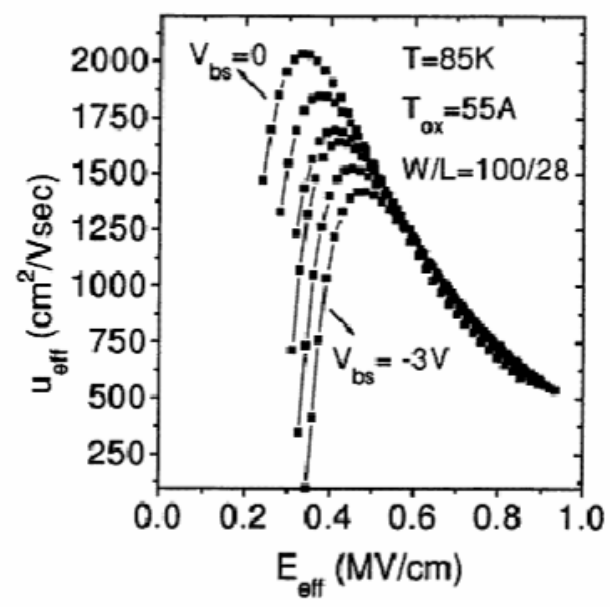

Figure 5. Measured effective carrier mobility ( eff) vs. effective electric field (Eeff) in MOSFET for different substrate body biases at $85 \mathrm{~K}$. (W=100 m, L=28 m) [26].

Again, as channel length is increased, carrier mobility is increased. Due to short channel effect (SCE) for short channel devices, carrier mobility becomes lower. But in case of CNTFET, the problem of SCE is not effective and there are no difficulties due to scattering. So, carrier mobility is independent of channel length. Moreover, bulk and interface charge impurity scattering dominates at low effective electric field and that is why carrier mobility is decreased. At high effective electric field (Eeff), surface roughness and photon scattering becomes effective and again effective carrier mobility (ueff) is degraded. In these cases, channel length of the MOSFET is kept constant. Figure 5 shows measured effective carrier mobility with various substrate body biases (Vbs) at $85 \mathrm{~K}$. At low temperature, mobility is separated for different Vbs and low Eeff as 
Columbic scatterings dominate at these regions. Larger Vbs leads to mobility, eff decreasing for same low Eeff due to reducing of electron density in the channel. As the scattering mechanisms are not dominant in CNTFET devices, so scattering hardly affects the carrier mobility $[24,26]$.

\subsection{Effect of short channel length and effective channel length}

Channel length of CNTFET is small compared to the MOSFET. At the shorter channel length, ballistic transport of carrier occurs in CNTFET and shows better performance. For CNTFET, as the channel length of device decreases, the performance of the device upgrades due to ballistic conduction. But in case of MOSFET device, as channel length decreases, short channel effect (SCE) becomes more dominant. A MOSFET device will have the problem of SCE when the order of the magnitude of the channel length is same as the depletion layer widths of the source and drain junction [1, 2]. Fig 6 (a) \& (b) shows the channel length scaling for different gate and drain voltage for MOSFET and CNTFET respectively [25, 28].

When an MOS device is in saturation, the effective channel length actually is decreased such that Leff $=\mathrm{L}-$ Lshort. Effective channel length depends upon different properties of materials such as capacitance, dielectric constant etc. Fig. 6 (a) shows that as drain voltage increases, the effective channel length decreases, for different types of dielectric materials. In case of CNTFET, the material properties may not reduce the effective channel length [2].

Again, for MOS devices, when channel length is reduced, the threshold voltage decreases. This is caused due to the decrease in potential barrier, which introduces Drain Induced Barrier Lowering (DIBL) [1]. For CNTFET, as there is no problem of short channel effect (SCE) like MOSFET, no shift of threshold voltage causes here. In case of MOS devices, due to SCE, as channel length decreases, there is a significant increase in leakage current and DIBL, which reduces the switching speed of device [2]. But in CNTFET, leakage current and DIBL is independent of effective channel length. Besides SCE problem, if the channel length is increased, parasitic resistance will be increased and MFP (mean free path) for scattering will also be increased. That is why drain current will be decreased with certain gate voltage (Fig. 6 (b)). But, channel length of CNTFET is smaller than MOSFET and that's why, problems due to parasitic resistance are more effective in MOSFET than CNTFET [27].

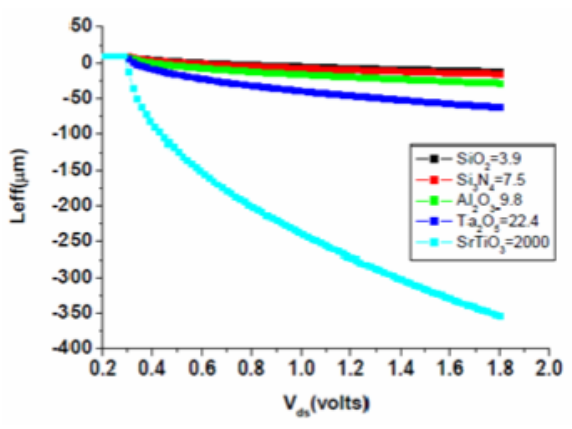

(a)

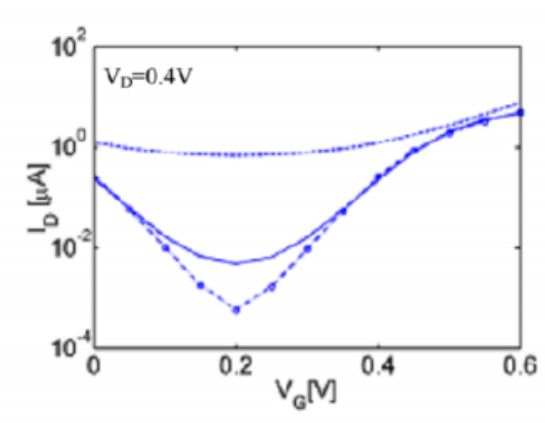

(b)

Figure 6 (a). Effective channel lengths vs Vds for various dielectric materials in MOSFET [25]. And (b). Channel length scaling according to characteristics graph of ID vs. VG of CNTFET. The channel length, $\mathrm{Lch}=15,10.5 \mathrm{~nm}$ and gate $\mathrm{ZrO} 2$ thickness $\mathrm{nm}$ tox $=2,2,1 \mathrm{~nm}$ (for dash-dot line, solid line and dashed line respectively) [28]. 
There is an optimization needed for fabricating CNTFET considering channel length as long channel length provides high carrier mobility where short channel length dominates in ballistic conduction which is not present in MOSFET.

\subsection{Transconductance variability due to gate capacitance and bias voltage}

If the transconductance of a CNTFET is gm, then it can be defined by this following equation [29]:

$$
g_{m}=\frac{\mu\left(\frac{c_{g g}}{L}\right)}{\left(\frac{V_{d s}}{L}\right)}
$$

Where, $\mathrm{C}_{\mathrm{gg}}$ is the gate capacitance, is the carrier mobility and $\mathrm{L}$ is the gate length. Here it can easily be found that the greater the carrier mobility and gate capacitance and lower the gate length, the higher the transconductance. For a CNTFET, and L are fixed and gate capacitance is defined by the equation [29]:

$$
C_{g g}=\frac{2 \pi \mathrm{K} \varepsilon_{0}}{\ln \left(\frac{4 t}{d}\right)}
$$

Where, $\mathrm{t}$ is the dielectric thickness and $\mathrm{d}$ is the diameter of CNT. For top/bottom gate CNT, is high because of its highly dielectric nature. High- value increases gate capacitance Cgg, which enlarge the transconductance gm value. On the other hand, for a SBCNTFET, lowering or elimination of the schottky barrier at the nano-tube metal surface by the high electric field at the dielectric metal surface, transconductance will be affected [29]. Effective gate capacitance will be found dividing gate capacitance by $2 \mathrm{~d}(\mathrm{~d}=\mathrm{CNT}$ diameter $)$. It is measured $\sim 5.5 \mathrm{Fcm}-2$ for CNTFET, which is better as compared to about 2.4 Fcm-2 for MOSFET [30].

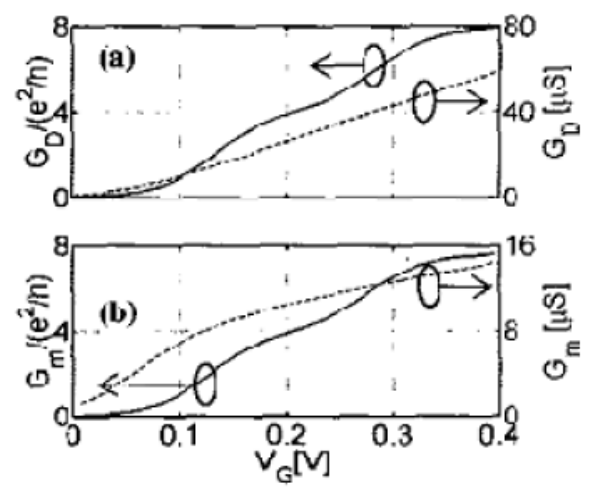

Figure 7 (a). the channel conductance and (b). transconductance vs. gate voltage in CNTFET when Cins= $0.4 \mathrm{pF} / \mathrm{cm}$ (dashed line in right axis) and Cins $=90 \mathrm{pF} / \mathrm{cm}$ (solid line in left axis) [31].

Fig. 7 (a) \& (b) shows the behaviors of channel conductance and transconductance as a function of gate voltage, VG for low and high insulator capacitance, Cins. In the quantum capacitance limit (high Cins), the channel conductance is quantized in units of $4 \mathrm{e} 2 / \mathrm{h}$. It is experimentally found that Fermi level is observed at the bottom of first sub-band with larger gate voltage showing quantization effect. That's why VG dominates in CNT potential [31]. 


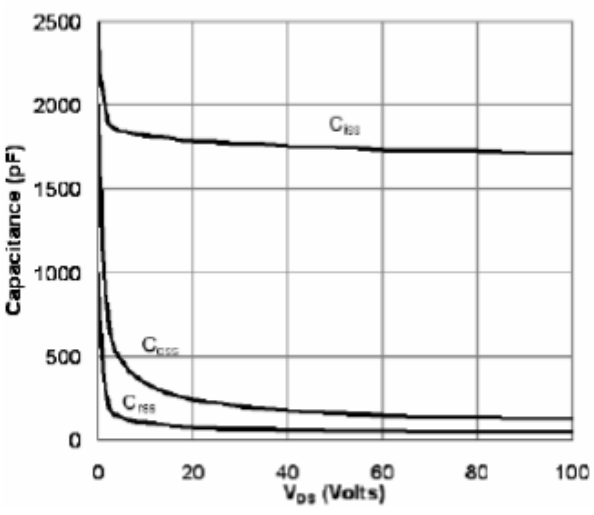

(a)

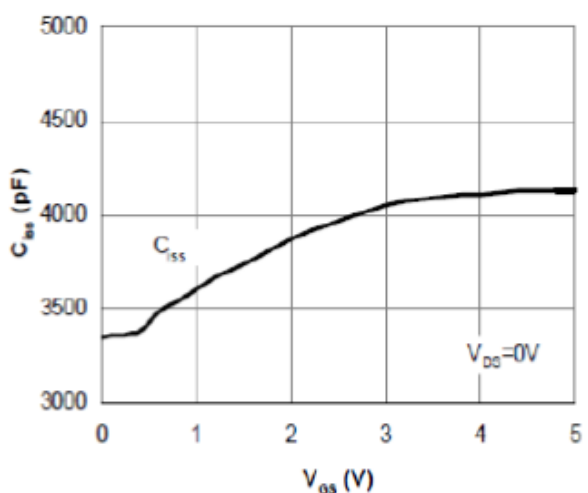

(b)

Figure 8 (a). Typical variation of Capacitances with VDS in MOSFET. (b) Typical variation of Capacitance with VGS in MOSFET [30, 32]

Moreover, the MOSFET capacitances are non-linear and a function of the dc bias voltage. Fig. 8 (a) shows how capacitances correspond with increased VDS voltage. The decrease in capacitances with VDS is caused by the decrease in depletion capacitance as the voltage increases and the depletion region widens. Fig. 8 (b) shows that when the VGS voltage increases past the threshold voltage (for low VDS values), the MOSFET gate capacitance also increases because of the formation of an inversion layer of electrons in the MOS channel and an accumulation layer of electrons under the trench bottom. This is why the slope of the gate charge curve is increased once the voltage goes beyond the Qgd phase [30, 32].

\subsection{Effect of contact resistance on switching capacity and drain current}

The contact resistance depends strongly on the atomic structure in the contact region when intermolecular nanotube junctions are formed. An interesting feature of this junction is the intuitive dependence of conductance on the contact length, 1. Fig. 9 (a) and Fig. 9 (b) shows the conductance values for armchair-armchair and zigzag-zigzag tube junctions respectively. It is also interesting to note that when the tubes are in-registry, the conductance values are high and comparable to ideal tubes. Therefore, this simple end-end contact geometry is an excellent way of connecting multiple tubes in device applications [5].

On the other side, when the nanotubes are displaced shortly from in-registry configurations, then the conductivity of the inner tubes gets reduced huge significantly. Thus, rapid switching between high and low conductance states could be achieved and fast atomic scale switches can be structured by using these end-end junctions. It is also found that the contact resistance can vary several orders of magnitude with atomic scale movement. This modulation of contact resistance improves the switching speed of CNTFET. In MOSFET, altering channel resistance affects switching capacity [5]. 

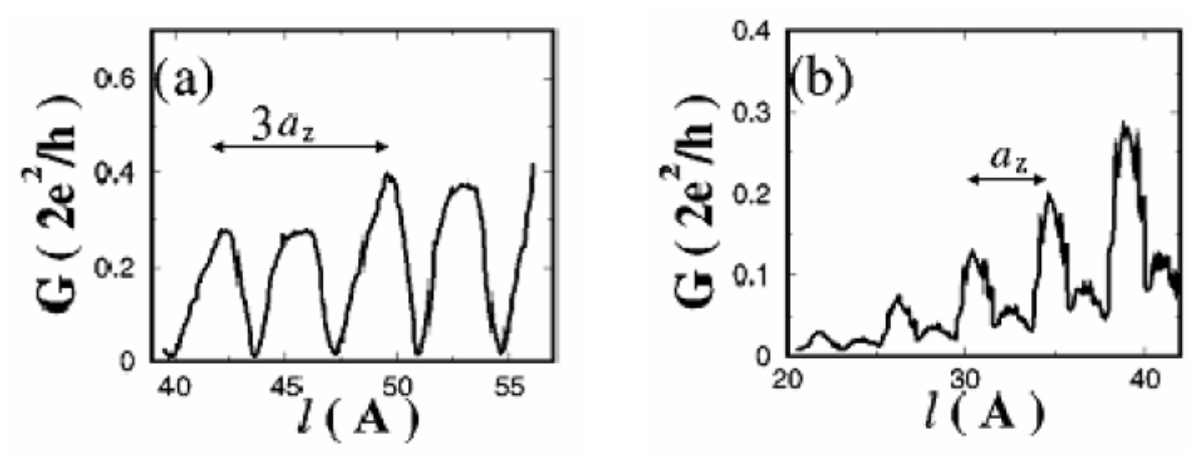

Figure 9 (a). Variation of conductance at the Fermi energy as a function of contact length, 1 for the (10, 10)$(10,10)$ junction (Armchair CNT) where unit cell length $a z=2.46 \AA$. (b). Variation of conductance with 1 for the $(18,0)-(18,0)$ junction (Zigzag CNT) where az $=4.26 \AA$ [5].

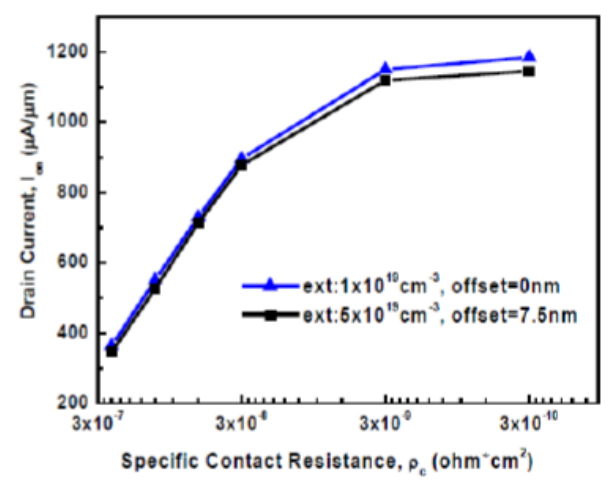

Figure 10. Relationship between Ion and specific contact resistance $\rho \mathrm{c}$ in $45 \mathrm{~nm}$ NMOS device (contact area $=0.045 \mathrm{mx} 1 \mathrm{~m})[33]$

Again, MOSFET channel resistance is reduced with scaled channel length, but on the other hand, the accordingly scaled contact area causes an increment in contact resistance if specific contact resistance is not scaled. Fig. 10 shows that technology to reduce specific contact resistance has a major impact on drain current as well as transconductance in deep sub-micron MOSFET [33].

\subsection{Ballistic conduction and increased charge density}

CNT shows ballistic conduction at room temperature. For SWCNT, it is $200 \mathrm{~nm}$ scale. For MWCNT, both diffusive and ballistic properties are found. MOSFET does not show ballistic conduction because gate voltage of MOSFET holds some charge in the channel at the beginning $[14,31]$. 


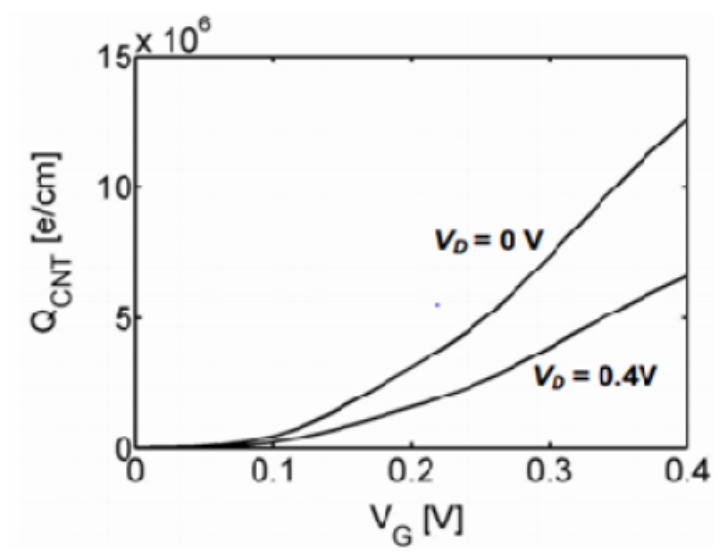

Figure 11. The charge density vs. gate voltage curve for CNT [34]

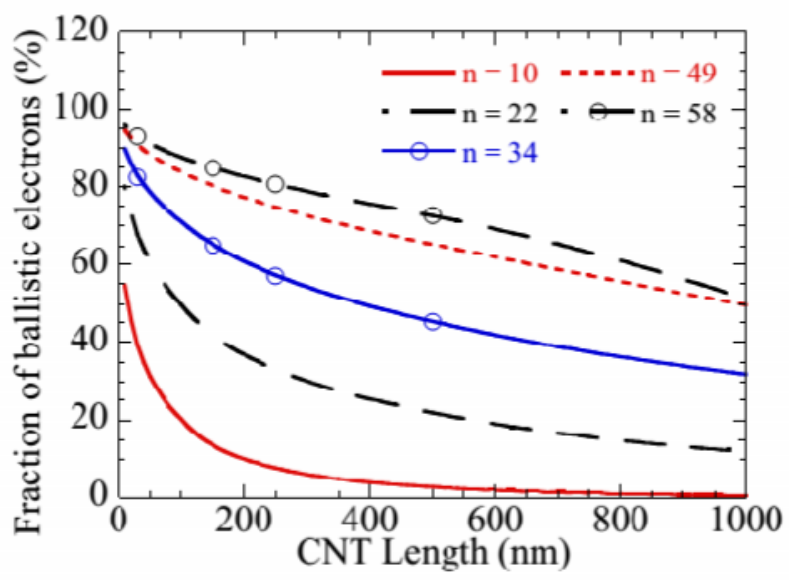

Fig. 12. Fraction of ballistic electrons (\%) as a function of CNT length $(\mathrm{E}=1 \mathrm{kV} / \mathrm{cm})[20]$.

As ballistic conduction is non-resistive and scattering mechanism can be ignored, then current density is higher in CNTFET than MOSFET. Fig. 11 shows the variation of charge density (QCNT) at the beginning of the channel with increasing gate voltage (VG). Gate voltage induces charges at the channel. As the gate voltage increased, the source-drain barrier lowers and then current flows between source and drain due to the flow of charge. If gate voltage is increased, more carriers flow through the channel due to ballistic conduction and so charge density in channel is increased which increases drain current. But in case of MOSFET, ballistic conduction is not possible and that is why ON current does not increase significantly with increasing gate voltage because charge density does not rise like CNTFET with varying gate voltage due to absence of ballistic conduction [3, 34].

Number of ballistic electrons are dependent on CNT length with low field step of $1 \mathrm{kV} / \mathrm{cm}$ shown in Fig. 12. For this low field regime, the main scattering event corresponds to electron-photon acoustic scattering. For lager tubes, 50\% electrons are ballistic (ballistic curve is decreased slowly) and for smaller tubes, the electron ballisticity is reduced at first 200-300 nm, then going down almost in constant manner [20]. 


\section{CONCLUSION}

In recent future, fabrication of a single IC where transistors extending in three dimensions is called 3D VLSI technology, which could be the future of IC fabrication emerging the size: from tiny to tinier. But, it is restricted by the physical limits to transistor scaling due to source-to-drain leakage, limited gate metals and limited options for channel material. CNTFET could be an appropriate device for its small size and exclusive ballistic, heat dissipation and low resistive properties. In this study, various existing types of CNTFET and its distinct properties over MOSFET have been reviewed. Due to high dielectric material, mobility of carrier increases whereas channel dimension decreases rigorously. The special property of channel dimension leads to ballistic conduction due to large density in channel, presenting CNTFET as more suitable in nanoelectronic devices than MOSFET [35]. In addition, semiconductor memory devices can be fabricated by using CNTFET for its high on/off current will open a new era in nano-computing. This has to be mentioned that manufacturing by CNTFET is quite costly and CNTFET has lower reliability due to growth of misaligned CNTs in growth process and increasing leakage power and noise margin in metallic CNT. Also, manufacturing variation in doping, densities of tubes, static noise could increase the delay in functioning power [36]. Considering all of these, CNTFET becomes one of the most promising candidates for Si MOS technology and its stunning performance opens a door of new possibilities in the field of nanotechnology.

\section{REFERENCES}

[1]F. D'Agostino and D. Quercia, "Short Channel Effects in MOSFETs", Introduction to VLSI design (EECS 467), 2001.

[2]D. Rechem, S. Latreche and C. Gontrand, "Channel length scaling and the impact of metal gate work function on the performance of double gate-metal oxide semiconductor field-effect transistors", PramanaJournal of Physics, Indian Academy of Sciences, Vol. 72, No. 3, pp. 587-599, 2009.

[3]P. Desgreys, J. G. D. Silva and D. Robert, "Dispersion Impact on Ballistic CNTFET N+-I-N+ Performances", Dans European Nano Systems Workshop - ENS 2006.

[4]P. Poncharal, C. Berger, Y. Yi, Z. Wang and W. A de Heer, "Room temperature ballistic conduction in carbon nanotubes", Journal of Phy. Chem. B, Vol. 106, 12104, 2002.

[5]A. Buldum and J. P. Lu, "Contact Resistance between Carbon Nanotubes", Phy. Rev. B, Vol. 63, 161403(R).

[6]Ouyang, Yijian, Guo, Jing, "Heat dissipation in carbon nanotube transistors". Appl. Phy. Lett., vol. 89, No. 18, pp. 3122, 2006.

[7]M. Claus, S. Blawid, P. Sakalas and M. Schroter, "Analysis of the frequency dependent gate capacitance in CNTFETs", SISPAD 2012.

[8]A. H. Chowdhury, N, Akhter and A. A. Faisal, "Performance Analysis and Development of SelfConsistent Model of CNTFET", Proc. of the Glob. Engg, Sci. and Tech. Conf., Bangladesh, December 2012.

[9]R. Sahoo and R. R. Mishra, "Simulations of Carbon Nanotube Field Effect Transistor", International J.of Electrical Engg. Research, Vol. 1, No. 2, pp. 117-125, 2009.

[10]S. Heinze, J. Tersoff, R. Martel and V. Derycke, "Carbon Nanotubes as Schottky Barrier Transistors", Phy. Rev. Lett., Vol. 89, pp. 106801, 2002.

[11]Pourfath M., Kosina H. and Selberherr S., "Tunneling CNTFETs”, J. Comp. Electron, Vol. 6, pp. 243246, 2007.

[12]G. Pennington and N. Goldsman, "Semi classical transport and phonon scattering of electrons in semiconducting carbon nanotubes," Phy. Rev. B, vol. 68. 045426, pp. 1-11, 2003.

[13]Geim A. K. and Novoselov K. S., “The rise of Graphene”, Nat. Mat., Vol. 6, No. 3, pp. 183-191, 2007.

[14]Z. Zhang, "Carbon Nanotube Electronics", Royal Institute of Technology, KTH, 04-2009. 
[15]Dekker, C. "Carbon nanotubes as molecular quantum wires", Physics Today, Vol. 52, No. 5, pp. 22-28, 1999.

[16]Mintmire J. W., Dunhap B. I. and White C. T., “Are fullerene tubes metallic?”, Phy. Rev. Lett., Vol. 68. No. 5. Pp. 631-634, 1992.

[17]Hasina F. Huq et al., "Study of Carbon NanoTube Field Effect Transistors for NEMS", InTech, ISBN: 978-953-307-054-4, March, 2010.

[18]Shanghai Jiao Tong, "Muti-channeled carbon nanotube field effect transistor (MC-CNTFET)", Res. Inst. of Micro/Nano Sci. and Tech., University, HuJiao ICP 2010869.

[19]C. J. Först, C. R. Ashman, K. Schwarz and P. E. Blöchl, "The interface between silicon and a high-k oxide", Nature 427, 53, 2004.

[20]H. Cazin d'Honincthun et al., "Monte Carlo study of coaxially gated CNTFETs: capacitive effects and dynamic performance", Comptes Rendus Physique, Vol. 9, No. 1, pp. 67-77, 2008.

[21]Bipul C Paul, Shinobu Fujita, Masaki Okajima, and Thomas Lee, "Modeling and Analysis of Circuit Performance of Ballistic CNFET", Proc. of the 43rd Design Automation Conf., DAC 2006, San Francisco, USA, DOI: 10.1145/1146909.1147092.

[22]Dang T., Anghel L. and Leveugle R., "CNTFET Basics and simulation", International Conference on Design and Test of Integrated Systems in Nano Scale Technology, IEEE, doi: 10.1109/DTIS.2006.1708731, pp. 28 - 33, 2006.

[23]A. Chaudhry, "Mobility Models for Unstrained and Strained Silicon MOSFETS: A Review", Contemporary Engineering Sciences, Vol. 4, no. 5, pp. 229 - 247, 2011.

[24]K. Chainy, J. Huangz, J. Dustery, P. K. Kox and C. Huy, "A MOSFET electron mobility model of wide temperature range (77-400 K) for IC simulation", Semicond. Sci. Tech. 12, pp. 355-358, UK PII: S02681242(97)78841-2, 1997.

[25]K. Niranjan, S. Srivastava, J. Singh and M. Tiwari "Comparative Study: MOSFET and CNTFET and the Effect of Length Modulation", Int. J. of Rec. Tech. and Engg. (IJRTE), Vol. 1, No. 4, 2012.

[26]K. Chain et al., "A MOSFET electron mobility model of wide temperature range (77-400 K) for IC simulation”, Semicond. Sci. Technol., Vol. 12, pp. 355-358. 1997.

[27]A. Javey et al., "Carbon Nanotube Field effect Transistors with Integrated Ohmic Contacts and High-k gate dielectrics", Nano Letters, Vol. 4, No. 3, 447-450, 2004.

[28]J. Guo et al., "A numerical study of scaling issues for Schottky-barrier carbon nanotube transistors", Electron Devices, IEEE Transaction, Vol .51, no. 2, pp. 172-177, Feb. 2004.

[29]M. H. Yang et al., "Advantages of top-gate, high-k dielectric Carbon nanotube field-effect transistors", Applied Physics Letters 88, 113507, 2006.

[30]Alpha \& Omega Semiconductor, "Power MOSFET Basics", pp. 1-11. url: http://www.aosmd.com.

[31]J. Guo et al., "Assessment of Silicon MOS and Carbon Nanotube FET Performance Limits Using a General Theory of Ballistic Transistor". Electron Devices Meeting, 2002. IEDM '02. International, pp. 711 - 714. [32] B. J. Baliga, "Fundamentals of Power Semiconductor Devices", pp. 385-390, SpringerScientific, New York, 2008.

[33]J. Yuan, P. M. Zeitzoff and J. C. S. Woo, "Source/Drain Parasitic Resistance Role and Electrical Coupling Effect in sub 50nm MOSFET Design", ESSDERC, 2002.

[34]A. Rahman, J. Guo, S. Datta, and M. Lundstrom, "Theory of Ballistic Nanotransistors", IEEE Transactions on Electron Devices, Vol. 50, No. 9, 2003.

[35]J. Guo, A. Javey, H. Dai, and M. Lundstrom, "Performance analysis and design optimization of near ballistic carbon nanotube FETs," IEDM tech. digest, pp. 703-706, 2004.

[36]Zhen Wang, Mark Karpovsky and Ajay Joshi, "Influence of Metallic Tubes on the Reliability of CNTFET SRAMs: Error Mechanism and Countermeasures", Great Lakes Symposium on VLSI 2011, Lausanne, Switzerland. 